\title{
Patterns of co-morbidity with anxiety disorders in Chinese women with recurrent major depression
}

\author{
Y. $\mathrm{Li}^{1}$, S. Shi ${ }^{2,3}$, F. Yang ${ }^{3}$, J. Gao ${ }^{4}$, Youhui $\mathrm{Li}^{5}$, M. Tao ${ }^{6}$, G. Wang ${ }^{7}$, K. Zhang ${ }^{8}$, C. Gao ${ }^{9}$, L. Liu ${ }^{10}$, Kan Li $^{11}$, \\ Keqing Li ${ }^{12}$, Y. Liu ${ }^{13}$, Xumei Wang ${ }^{14}$, J. Zhang ${ }^{15}$, L. Lv ${ }^{16}$, Xueyi Wang ${ }^{17}$, Q. Chen ${ }^{18}$, J. Hu ${ }^{19}$, L. Sun ${ }^{20}$, \\ J. Shi ${ }^{21}$, Y. Chen ${ }^{22}$, D. Xie ${ }^{1}$, J. Flint ${ }^{1}$, K. S. Kendler ${ }^{2 *}$ and Z. Zhang ${ }^{2 *}$ \\ ${ }^{1}$ Wellcome Trust Center for Human Genetics, Roosevelt Drive, Oxford, UK; ${ }^{2}$ Fudan University Affiliated Huashan Hospital, Shanghai, People's \\ Republic of China ; ${ }^{3}$ Shanghai Jiao Tong University School of Medicine Affiliated Shanghai Mental Health Center, Shanghai, People's Republic of \\ China ; ${ }^{4}$ Zhejiang Traditional Chinese Medical Hospital, Hangzhou, Zhejiang, People's Republic of China ; ${ }^{5}$ No. 1 Hospital of Zhengzhou \\ University, Zhengzhou, Henan, People's Republic of China ; ${ }^{6}$ Second Affiliated Hospital of Zhejiang Chinese Medical University, Hangzhou, \\ Zhejiang, People's Republic of China ; ${ }^{7}$ Beijing Anding Hospital, Capital Medical University, Xicheng District, Beijing, People's Republic of \\ China; ${ }^{8}$ No. 1 Hospital of Shanxi Medical University, Taiyuan, Shanxi, People's Republic of China ; ${ }^{9}$ No. 1 Hospital of Medical College of Xian \\ Jiaotong University, Xi'an, Shaanxi, People's Republic of China; ${ }^{10}$ Shandong Mental Health Center, Jinan, Shandong, People's Republic of China ; \\ ${ }^{11}$ Mental Hospital of Jiangxi Province, Nanchang, Jiangxi, People's Republic of China; ${ }^{12}$ Hebei Mental Health Center, Baoding, Hebei, People's \\ Republic of China $;{ }^{13}$ The First Hospital of China Medical University, He Ping District, Shenyang, Liaoning, People's Republic of China; \\ ${ }^{14}$ ShengJing Hospital of China Medical University, Shenyang, Liaoning, People's Republic of China; ${ }^{15}$ No. 3 Affiliated Hospital of Sun Yat-sen \\ University, Tian He District, Guangzhou, Guangdong, People's Republic of China; ${ }^{16}$ Psychiatric Hospital of Henan Province, No. 388 Jian She \\ Zhong Lu, Xinxiang, Henan, 453002, People's Republic of China ; ${ }^{17}$ The First Hospital of Hebei Medical University, Shijiazhuang, Hebei, People's \\ Republic of China; ${ }^{18}$ Dalian No. 7 People's Hospital and Dalian Mental Health Center, Gan Jing Zi District, Dalian, Liaoning, People's Republic \\ of China; ${ }^{19}$ No. 1 Mental Health Center Affiliated Harbin Medical University, Nangang District, Harbin, Heilongjiang, People's Republic of \\ China; ${ }^{20}$ Jilin Brain Hospital, Siping, Jilin, People's Republic of China ; ${ }^{21}$ Xi'an Mental Health Center, Qujiang Xin District, Xi'an, Shaanxi, \\ People's Republic of China; ${ }^{22}$ Clinical Trial Service Unit, Richard Doll Building, Roosevelt Drive, Oxford, UK; ${ }^{23}$ Virginia Institute for \\ Psychiatric and Behavioral Genetics of Virginia Commonwealth University, Richmond, VA, USA; ${ }^{24}$ No. 4 Affiliated Hospital of Jiangsu \\ University, Zhenjiang, Jiangsu, People's Republic of China
}

Background. Studies conducted in Europe and the USA have shown that co-morbidity between major depressive disorder (MDD) and anxiety disorders is associated with various MDD-related features, including clinical symptoms, degree of familial aggregation and socio-economic status. However, few studies have investigated whether these patterns of association vary across different co-morbid anxiety disorders. Here, using a large cohort of Chinese women with recurrent MDD, we examine the prevalence and associated clinical features of co-morbid anxiety disorders.

Method. A total of 1970 female Chinese MDD patients with or without seven co-morbid anxiety disorders [including generalized anxiety disorder (GAD), panic disorder, and five phobia subtypes] were ascertained in the CONVERGE study. Generalized linear models were used to model association between co-morbid anxiety disorders and various MDD features.

Results. The lifetime prevalence rate for any type of co-morbid anxiety disorder is $60.2 \%$. Panic and social phobia significantly predict an increased family history of MDD. GAD and animal phobia predict an earlier onset of MDD and a higher number of MDD episodes, respectively. Panic and GAD predict a higher number of DSM-IV diagnostic criteria. GAD and blood-injury phobia are both significantly associated with suicidal attempt with opposite effects. All seven co-morbid anxiety disorders predict higher neuroticism.

Conclusions. Patterns of co-morbidity between MDD and anxiety are consistent with findings from the US and European studies; the seven co-morbid anxiety disorders are heterogeneous when tested for association with various MDD features.

Received 6 August 2011; Revised 17 October 2011; Accepted 31 October 2011; First published online 30 November 2011

Key words: Co-morbid anxiety disorders, major depression.

\footnotetext{
* Address for correspondence: K. S. Kendler, M.D., Virginia Institute for Psychiatric and Behavioral Genetics of Virginia Commonwealth University, Box 980126, Richmond, VA 232980126, USA.

(Email: kendler@vcu.edu) [K.S.K.]

(Email: zhangzhen601020@yahoo.com.cn) [Z. Zhang]

The online version of this article is published within an Open Access environment subject to the conditions of the Creative Commons Attribution-NonCommercial-ShareAlike licence <http://creativecommons.org/licenses/by-nc-sa/2.5/>. The written permission of Cambridge University Press must be obtained for commercial re-use.
} 


\section{Introduction}

A large number of studies agree that anxiety disorders occur in patients with major depressive disorder (MDD) far more frequently than expected by chance (Kessler et al. 1996; Gorwood, 2004). In fact, in clinical settings, more than half of MDD patients have comorbid anxiety disorders (Zimmerman \& Chelminski, 2003). Furthermore, a number of clinical features characterize MDD patients with co-morbid anxiety. Typically, their depressive disorder is more severe, with more episodes, earlier onset age, greater suicidal risk and poorer treatment response (Brown et al. 2001); they have a lower social competence (Cerdá et al. 2010); and they are more likely to have a family history of MDD (Clayton et al. 1991; Kendler et al. 1992).

A number of explanations have been put forward to explain this co-morbidity. In part, it appears to arise from a shared genetic propensity to both anxiety and depression: multivariate twin modeling has identified a common genetic factor contributing to internalizing disorders, including MDD, generalized anxiety disorder (GAD) and phobias (Kendler et al. 2003). However, a common genetic origin is clearly not the whole explanation. Genetic studies themselves indicate that there are some genetic factors that act specifically on anxiety and MDD (Kendler et al. 2003). They also show that the genetic factors explain only part of the co-occurrence of illnesses. We know that non-shared environment explains almost all the covariance between MDD and agoraphobia and more than $40 \%$ between MDD and GAD for female twins (Cerdá et al. 2010). Our understanding of the nongenetic factors that drive co-morbidity is still very limited. The responsible environmental factors remain to be identified but it has been shown that low socioeconomic status in childhood and adult unemployment distinguishes the MDD GAD co-morbid group from the MDD-only group (Cerdá et al. 2010).

Studies that investigate the prevalence and features of MDD associated with co-morbid anxiety typically examine the relationship between MDD and a single anxiety disorder (such as GAD or panic disorder) (Garvey et al. 1987; Reich et al. 1993; Grunhaus et al. 1994; Maier et al. 1995; Kessler et al. 1998, 1999; Gorwood, 2004; Kendler et al. 2007). A few studies (Pini et al. 1997; Fava et al. 2000; Lamers et al. 2011) report that there are differences in the prevalence rates of co-morbid anxiety disorders, but rarely distinguish or control for different co-morbid anxiety disorders when testing for association with MDD-related features (Kessler et al. 2003; Fava et al. 2004; Rhebergen et al. 2011). This leaves open an important question: is there similarity in the clinical features of patients with MDD with different co-morbid anxiety disorders?
One possibility is that co-morbidity arises from separate and independent associations with each anxiety disorder, as reflected in the pattern of associations with symptoms, severity indices and risk factors. The alternative is that co-morbidity reflects a common mechanism among all anxiety disorders.

In this article, we examine co-morbid anxiety in a large cohort of female Han Chinese recruited through clinical settings and diagnosed with recurrent MDD using a structured clinical interview. We set out to determine first whether co-morbidity between anxiety and depression was similar in China to that observed elsewhere in the world. Our information about comorbid anxiety disorders, and the attendant clinical pattern, is currently derived almost exclusively from studies of European and US populations. To our knowledge, no one has examined whether the same pattern of co-morbidity holds true for MDD in China. Second, we asked how similar were the clinical features of MDD patients with different anxiety disorders, by comparing differences in association between clinical features of MDD and different comorbid anxiety disorders. Distinguishing the clinical features of different types of anxiety disorders helps us to identify risk factors that are associated with each anxiety disorder specifically, which may in turn alter the ways patients with MDD who have comorbid anxiety disorders are assessed and the treatments they are offered.

\section{Study subjects}

Data for the present study were drawn from the ongoing China, Oxford and VCU Experimental Research on Genetic Epidemiology (CONVERGE) study of MDD. The analysis was based on a total of 1970 cases recruited from 51 mental health centers and psychiatric departments of general medical hospitals in 40 cities in 21 provinces.

The project utilized samples collected for a genomewide association study of MDD. Given evidence that the genetic effects on MDD are different between men and women (Kendler et al. 2001), in order to control for this known genetic heterogeneity, we collected female participants only with four Han Chinese grandparents. Cases were excluded if they had a preexisting history of bipolar disorder, any type of psychosis or mental retardation. Cases were aged between 30 and 60 years, had two or more episodes of MDD with the first episode occurring between the ages of 14 and 50 years, and had not abused drugs or alcohol before their first episode.

All subjects were interviewed using a computerized assessment system, which lasted on average $2 \mathrm{~h}$. All interviewers, who were postgraduate medical 
students, junior psychiatrists or senior nurses, were trained by the CONVERGE team for a minimum of 1 week. The interview includes assessment of psychopathology, demographic and personal characteristics, and psychosocial functioning. Interviews were tape-recorded and a proportion of them were listened to by the trained editors, who provided feedback on their quality. The study protocol was approved centrally by the Ethical Review Board of Oxford University and the ethics committee in participating hospitals in China.

\section{Measures}

The diagnoses of depressive (dysthymia and MDD) and anxiety disorders (GAD, panic disorder with or without agoraphobia) were established with the Composite International Diagnostic Interview (World Health Organization lifetime version 2.1; Chinese version), which classifies diagnoses according to the Diagnostic and Statistical Manual of Mental Disorders, fourth edition (DSM-IV) criteria. The interview was originally translated into Mandarin by a team of psychiatrists in Shanghai Mental Health Center, with the translation reviewed and modified by members of the CONVERGE team. Phobias, divided into five subtypes (animal, situational, social and blood-injury, and agoraphobia), were diagnosed using an adaptation of DSM-IV criterion D (APA, 1994) requiring one or more unreasonable fears, including fears of different animals, social phobia and agoraphobia, that objectively interfered with the respondents' life or resulted in severe anxiety when exposed to the phobic stimulus. The section on the assessment of phobias was translated by the CONVERGE team from the interview used in the Virginia Adult Twin Study of Psychiatric and Substance Use Disorders (VATSPUD) (Kendler \& Prescott, 2006). The history of lifetime major depression in the parents and siblings was assessed using the Family History Research Diagnostic criteria (Endicott et al. 1975). Neuroticism was measured with the 23-item Eysenck Personality Questionnaire (Eysenck \& Eysenck, 1975).

All interview sections were fully computerized into a bilingual system of Mandarin and English developed in-house in Oxford, UK, called SysQ (a selfadministered interview system). Skip patterns were built into SysQ. Interviews were administered by trained interviewers and entered offline in real time onto SysQ, which was installed in laptop computers. Once an interview was completed, a backup file containing all the previously entered interview data could be generated with a database-compatible format. The backup file together with an audio record of the entire interview was uploaded to a designated server currently maintained in Beijing by a service provider. All the uploaded files in the Beijing server were then transferred to an Oxford server quarterly.

The diagnoses of MDD were based on information collected in the medical records and DSM-IV symptoms during the worst episode. Symptoms information collected for the diagnoses of co-morbid disorders of MDD was for lifetime.

\section{Statistical methods: generalized linear regression model choosing, analysis of variance (ANOVA)}

In order to test whether co-morbid anxiety disorders significantly predict various MDD features, including family history, age of onset, number of episodes, number of DSM-IV diagnostic criteria, suicidal attempt and neuroticism score, generalized linear models (GLMs) with different link functions were chosen for modeling different types of dependent variables controlling for age. Data for the age of onset were tested for normality and modeled with linear regression, and results represented as the standardized parameter estimate $(\beta)$. Suicidal attempt was modeled with logistic regression and results represented as odds ratios (ORs). The family history variable, represented by the total number of firstdegree family members among the two parents and all the siblings with a history of MDD, could be considered as a series of Bernoulli trials with a finite number of trials in statistical terms; hence binomial regression was used for modeling the association, weighted against the total number of family members for each subject. The same reason and choice were applied to variables such as the number of DSM-IV diagnostic criteria and the neuroticism score, which could also be considered as a finite number of Bernoulli trials. Poisson regression was chosen to model the number of episodes data, as it is considered to be count data with an upper limit of 96 (denoting 96 or more) being removed. Since binomial and Poisson regressions model the natural logarithms of the count and the event rate, respectively, results are represented by count ratio (CR) and rate ratio (RR), both calculated by exponentiating the regression coefficients. The statistical tests were implemented using $\mathrm{R}$ (R Development Core Team, 2011), which allows the specification of quasibinomial and quasipoisson link functions. They differ from binomial and Poisson functions only in that the dispersion parameter is not fixed at 1 ; in other words, over-dispersion is included in the model, which controls for the over-reporting of false-positive results.

In addition to calculating the individual effects of the seven co-morbid anxieties, the method of Analysis of Deviance for GLM Fits (ANOVA) was used to 
Table 1. Top 13 combinations among the total of 128 possibilities with seven co-morbid anxiety disorders that could either be present or not present in every patient with major depressive disorder

\begin{tabular}{lrr}
\hline 128 possibilities & Number & $(\%)$ \\
\hline Top 13 combinations & & \\
No anxiety & 765 & \\
GAD only & 184 & $(16.20)$ \\
Animal phobia only & 100 & $(8.80)$ \\
Blood-injury phobia only & 58 & $(5.11)$ \\
Situational phobia only & 45 & $(3.96)$ \\
GAD and animal phobia & 40 & $(3.52)$ \\
Animal and blood-injury phobia & 33 & $(2.90)$ \\
Animal and situational phobia & 29 & $(2.55)$ \\
Panic only & 29 & $(2.55)$ \\
Animal and situational and & 27 & $(2.38)$ \\
$\quad$ blood-injury phobia & & \\
Social phobia only & 26 & $(2.29)$ \\
GAD and blood-injury phobia & 24 & $(2.11)$ \\
Agoraphobia only & 20 & $(1.76)$ \\
Total of top 12 combinations & 615 & $(54.14)$ \\
(excluding the non-anxious group) & & \\
Total of other combinations & 521 & $(45.86)$ \\
(excluding the non-anxious group) & & \\
Total (excluding the non-anxious group) & 1136 & $(100.00)$ \\
& & \\
\end{tabular}

GAD, Generalized anxiety disorder.

examine whether the anxiety regression coefficients in the multiple GLMs were homogeneous or heterogeneous in predicting each of the MDD features. Two GLM objects were specified in ANOVA; one with a derived independent variable which is calculated as the sum of all seven anxiety diagnostic status, nested by another containing all the seven co-morbid anxiety diagnoses as additive independent variables. The result gives a table with a row for the residual degrees of freedom and deviance for each model. Test statistics could be specified in ANOVA, comparing the reduction in deviance for the row to the residuals. For models with dispersion estimated by moments, such as Gaussian, quasibinomial and quasipoisson fits, the $F$ test is considered to be the most appropriate.

\section{Results}

The lifetime prevalence rate in our sample of women with recurrent MDD for any of the seven co-morbid anxiety disorders was $60.2 \%$. The rates of the individual anxiety disorders in our cohort were $26.4 \%$ for GAD, $10.1 \%$ for panic disorder, $15.0 \%$ for agoraphobia, $14.3 \%$ for social phobia, $28.0 \%$ for animal phobia, $20.3 \%$ for situational phobia, and $21.7 \%$ for blood-injury phobia.
Table 2. Variables included in general linear models

\begin{tabular}{lrc}
\hline & Mean (s.D.) & NA \\
\hline Age, years & $45.11(8.84)$ & 16 \\
Age of onset, years & $35.87(9.97)$ & 45 \\
Number of episodes & $4.20(5.03)$ & 44 \\
$\quad$ (excluding 96 or more $^{\text {a }}$ & & \\
Number of DSM-IV diagnostic criteria & $8.28(1.05)$ & 39 \\
Number of positively diagnosed & $0.49(0.83)$ & 53 \\
$\quad$ family members & & \\
Number of family members & $4.95(1.75)$ & 53 \\
Neuroticism & $12.72(5.84)$ & 55 \\
\hline
\end{tabular}

S.D., Standard deviation; NA, number of missing data entries; DSM, Diagnostic and Statistical Manual of Mental Disorders.

${ }^{\text {a }}$ See Statistical methods section.

The mean number of co-morbid anxiety disorders among the MDD patients was 1.35 (S.D. =1.56). Among the $1136 \mathrm{MDD}(59.8 \%)$ patients who had at least one of the seven co-morbid anxiety disorders, $59.3 \%$ patients had two or more co-morbid anxiety disorders. Of the seven anxiety disorders, there are 128 possible combinations of what a patient might or might not have. Table 1 contains the top 13 combinations including the $40.2 \%$ of MDD patients without any of the seven comorbid anxiety disorders, $16.2 \%$ with GAD only, $8.8 \%$ with animal phobia only, and $5.1 \%$ with blood-injury phobia only, etc. The top 12 combinations (excluding the non-anxious group) accounted for $54.1 \%$ of all the MDD cases with co-morbid anxiety disorders. Because so many patients had more than one condition, we used a multiple regression model in order to tease apart individual effects.

We looked at the relationship between clinical features of MDD and the seven anxiety disorders, attempting to identify those that were shared between disorders or were disorder-specific. Tables 2 and 3 contain the summary statistics of the variables included in the regression analysis. We first tested the effect of each disorder separately. Then we combined all disorders into one model to test for their joint effect on each MDD feature.

We found significant effects for each disorder when tested separately, as well as combining the seven diagnoses into one variable - any type of anxiety disorder (Table 4). Results for jointly testing disorders are more complex and are shown in Table 5. Panic and social phobia independently significantly predicted an increased family history of MDD, with social phobia showing the highest effect (CR 1.52, $p=5.58 \times 10^{-4}$ ), controlling for the additive effects of the other comorbid anxiety disorders. GAD with one or more 
Table 3. Suicide-related response rates and major depressive disorder prevalence rates for both parents

\begin{tabular}{lclccc}
\hline Items & $\begin{array}{l}\text { Positive } \\
\text { diagnoses }\end{array}$ & $\begin{array}{l}\text { Negative } \\
\text { diagnoses }\end{array}$ & Total & $(\%)$ & NA \\
\hline Suicide features & & & & & \\
$\quad$ Suicidal thought & 1196 & 739 & 1935 & $(61.81)$ & 35 \\
$\quad$ Suicidal plan & 830 & 1105 & 1935 & $(42.89)$ & 35 \\
$\quad \begin{array}{l}\text { Suicidal attempt } \\
\text { Family history }\end{array}$ & 423 & 1512 & 1935 & $(21.86)$ & 35 \\
$\quad$ Father & 192 & 1486 & 1678 & $(11.44)$ & 292 \\
$\quad$ Mother & 301 & 1427 & 1728 & $(17.42)$ & 242 \\
\hline
\end{tabular}

NA, Number of missing data entries.

Table 4. Effects of generalized linear models testing for association between each co-morbid anxiety disorder as well as any of the seven co-morbid anxiety disorders, and major depressive disorder features taken one at a time ${ }^{\mathrm{a}}$

\begin{tabular}{llrllll}
\hline $\begin{array}{l}\text { Anxiety } \\
\text { disorder }\end{array}$ & $\mathrm{FH}^{\mathrm{b}}$ & $\mathrm{AAO}^{\mathrm{c}}$ & $\mathrm{NE}^{\mathrm{d}}$ & $\mathrm{NDC}^{\mathrm{b}}$ & $\mathrm{SA}^{\mathrm{e}}$ & $\mathrm{N}^{\mathrm{b}}$ \\
\hline Panic & $1.53^{* * *}$ & $0.01^{\dagger}$ & $1.16 \dagger \dagger$ & $1.83^{* * *}$ & $0.80^{*}$ & $1.63^{* * *}$ \\
GAD & $1.26^{*}$ & $-0.04^{*}$ & $1.08^{\dagger}$ & $1.85^{* * *}$ & $1.53^{* * *}$ & $1.84^{* * *}$ \\
Agoraphobia & $1.63^{* * *}$ & $-0.01 \dagger$ & $1.13 \dagger \dagger$ & $1.58^{* * *}$ & $1.38^{* * *}$ & $1.71^{* * *}$ \\
Social phobia & $1.88^{* * *}$ & $-0.02 \dagger$ & $1.18^{*}$ & $1.51^{* * *}$ & $1.35^{* * *}$ & $1.78^{* * *}$ \\
Animal phobia & $1.40^{* * *}$ & $-0.02 \dagger$ & $1.22^{* * *}$ & $1.35^{* * *}$ & $1.24^{* * *}$ & $1.56^{* * *}$ \\
Situational phobia & $1.46^{* * *}$ & $0.0{ }^{\dagger}$ & $1.11^{\dagger}$ & $1.33^{* * *}$ & $1.27^{* *}$ & $1.62^{* * *}$ \\
Blood-injury phobia & $1.50^{* * *}$ & $0.01^{\dagger}$ & $1.12 \dagger \dagger$ & $1.46^{* * *}$ & $0.84^{* *}$ & $1.56^{* * *}$ \\
Any & $1.56^{* * *}$ & $-0.04^{*}$ & $1.20^{* *}$ & $1.73^{* * *}$ & $1.33^{* * *}$ & $1.80^{* * *}$ \\
\hline
\end{tabular}

FH, Family history; AAO, age of onset; NE, number of episodes ; NDC, number of Diagnostic and Statistical Manual of Mental Disorders (DSM)-IV criteria ; SA, suicidal attempt; N, neuroticism; GAD, generalized anxiety disorder; OR, odds ratio; $\mathrm{CR}$, count ratio; $R R$, rate ratio.

${ }^{a}$ Values reported for the four types of regression models: linear regression: standardized parameter estimates $(\beta)$; logistic regression: OR; quasi-binomial regression: CR; quasi-Poisson regression: RR. ORs, CRs and RRs were calculated by exponentiating the regression coefficients.

${ }^{\mathrm{b}}$ Quasibinomial regression was used to model FH, NDC and N.

${ }^{\mathrm{c}}$ Linear regression was used to model AAO.

${ }^{\mathrm{d}}$ Quasipoisson regression was used to model NE.

${ }^{\mathrm{e}}$ Logistic regression was used to model SA.

${ }^{*} p<0.05,{ }^{* *} p<0.01,{ }^{* * *} p<0.001$.

$\dagger p<1, \dagger \uparrow p<0.1$.

than 1-month duration $\left(\beta=-0.04, p=4.71 \times 10^{-2}\right)$ significantly predicted earlier MDD onset age. Animal phobia (RR $1.18, p=1.12 \times 10^{-2}$ ) significantly predicted higher number of MDD episodes. Panic and GAD independently significantly predicted higher numbers of positive DSM-IV diagnostic criteria, with GAD $\left(\mathrm{CR} 1.70, p=3.62 \times 10^{-8}\right)$ showing the largest and most significant effect. GAD and blood-injury phobia were significantly associated with the probability of a suicide attempt, with GAD predicting higher probability
(OR 1.38, $p=9.95 \times 10^{-3}$ ), and blood-injury phobia predicting lower probability (OR $0.66, p=9.35 \times 10^{-3}$ ). All anxiety disorders independently and significantly predicted a higher neuroticism score, with GAD (CR 1.69, $p=7.42 \times 10^{-22}$ ) showing the highest effect.

We looked for common patterns in the way that anxiety disorders made an impact on the clinical features of MDD. Our hypothesis was that we would find some clinical features on which one or more anxiety disorder had similar impacts, as assessed 
Table 5. Effects of multivariate generalized linear models testing for association between the seven co-morbid anxiety disorders all considered together and various major depressive disorder features ${ }^{\mathrm{a}}$

\begin{tabular}{llccccc}
\hline Anxiety disorder & $\mathrm{FH}^{\mathrm{b}}$ & $\mathrm{AAO}^{\mathrm{c}}$ & $\mathrm{NE}^{\mathrm{d}}$ & $\mathrm{NDC}^{\mathrm{b}}$ & $\mathrm{SA}^{\mathrm{e}}$ & $\mathrm{N}^{\mathrm{b}}$ \\
\hline Panic & $1.32^{*}$ & $0.01 \dagger$ & $1.10 \dagger$ & $1.58^{* *}$ & $0.78 \dagger$ & $1.31^{* *}$ \\
$\mathrm{GAD}$ & $1.11 \dagger$ & $-0.04^{*}$ & $1.04 \dagger$ & $1.70^{* * *}$ & $1.38^{* *}$ & $1.69^{* * *}$ \\
Agoraphobia & $1.16 \dagger$ & $-0.01 \dagger$ & $1.01 \dagger$ & $1.18 \dagger$ & $1.25 \dagger$ & $1.17^{*}$ \\
Social phobia & $1.52^{* * *}$ & $-0.02 \dagger$ & $1.09 \dagger$ & $1.15 \dagger$ & $1.18^{\dagger}$ & $1.29^{* * *}$ \\
Animal phobia & $1.10 \dagger$ & $-0.02 \dagger$ & $1.18^{*}$ & $1.08 \dagger$ & $1.12 \dagger$ & $1.18^{* *}$ \\
Situational phobia & $1.06 \dagger$ & $0.00 \dagger$ & $0.99 \dagger$ & $1.03 \dagger$ & $1.24 \dagger$ & $1.20^{* *}$ \\
Blood-injury phobia & $1.21 \dagger \dagger$ & $0.03 \dagger$ & $1.03 \dagger$ & $1.22 \dagger \dagger$ & $0.66^{* *}$ & $1.19^{* *}$ \\
Over-dispersion parameter & $1.51 \dagger$ & - & $5.58 \dagger$ & $1.61 \dagger$ & - & $5.32 \dagger$ \\
\hline
\end{tabular}

FH, Family history; AAO, age of onset; NE, number of episodes; NDC, number of Diagnostic and Statistical Manual of Mental Disorders (DSM)-IV criteria; SA, suicidal attempt; N, neuroticism; GAD, generalized anxiety disorder; OR, odds ratio; $\mathrm{CR}$, count ratio; RR, rate ratio.

${ }^{a}$ Values reported for the four types of regression models: linear regression: standardized parameter estimates $(\beta)$; logistic regression: OR; quasi-binomial regression: CR; quasi-Poisson regression: RR. ORs, CRs and RRs were calculated by exponentiating the regression coefficients.

${ }^{\mathrm{b}}$ Quasibinomial regression was used to model FH, NDC and N.

${ }^{\mathrm{c}}$ Linear regression was used to model AAO.

${ }^{\mathrm{d}}$ Quasipoisson regression was used to model NE.

${ }^{\mathrm{e}}$ Logistic regression was used to model SA.

${ }^{\mathrm{f}}$ The over-dispersion parameter was included in the model due to the quasi-terms.

${ }^{*} p<0.05, * * p<0.01, * * * p<0.001$.

$\dagger p<1, \dagger \uparrow p<0.1$.

Table 6. ANOVA F test examining the heterogeneity of the multiple regression coefficients of the seven co-morbid anxiety disorders in predicting each of the six major depressive disorder features

\begin{tabular}{lllllll}
\hline Anxiety & FH & AAO & NE & NDC & SA & N \\
\hline ANOVA $F$ test & 1.04 & 1.32 & 0.59 & 3.48 & 2.79 & 5.72 \\
ANOVA $p$ value & 0.40 & 0.25 & 0.74 & $1.99 \times 10^{-3}$ & $1.02 \times 10^{-2}$ & $6.64 \times 10^{-6}$ \\
\hline
\end{tabular}

ANOVA, Analysis of variance; FH, family history ; AAO, age of onset; NE, number of episodes; NDC, number of Diagnostic and Statistical Manual of Mental Disorders (DSM)-IV criteria; SA, suicidal attempt; N, neuroticism.

by the equivalence of their regression coefficients. We applied a global heterogeneity test for the multiple regression coefficients. The results showed that coefficients of the seven co-morbid anxiety disorders were homogeneous with respect to their association with family history, age of onset and the number of episode data. However, they were heterogeneous with respect to their association with the number of DSM-IV diagnostic criteria $\left(F=3.48, p=1.99 \times 10^{-3}\right)$, suicidal attempt $\left(F=2.79, p=1.02 \times 10^{-2}\right)$ and neuroticism score $\left(F=5.72, p=6.64 \times 10^{-6}\right)$ (Table 6). When the diagnoses of all seven co-morbid anxiety disorders were included in a multiple regression model, controlling for each other's additive effects, the ones that were significant when tested for association with various MDD features were different, such that only GAD predicted earlier MDD onset, whereas only animal phobia predicted more episodes of MDD. Results from global heterogeneity tests also indicated that MDD patients with different co-morbid anxiety disorders manifested different clinical features.

\section{Discussion}

Our work establishes two important findings: first, in China co-morbidity between MDD and anxiety disorders is similar to that reported in the West; second, there is important heterogeneity in the association between anxiety disorders and some clinical features of MDD. We discuss these two points below.

The lifetime prevalence rate for any type of comorbid anxiety disorder among MDD patients recruited through clinical settings is $60.2 \%$. This is higher than the $50.6 \%$ lifetime rate reported in the Depression Research Program with patients recruited through clinical settings (Fava et al. 2000), and lower 
than the $75 \%$ lifetime rate reported in the Netherlands Study of Depression and Anxiety (NESDA) with patients mostly recruited through primary care (54\%) and specialized mental health settings (27\%) (Lamers et al. 2011). Additionally, the reported lifetime co-morbidity rates for panic disorder among MDD patients in a cross-national epidemiological survey study are $10.7 \%$ in the USA, $16.2 \%$ in West Germany, $17 \%$ in Korea and $5.9 \%$ in Taiwan (Weissman et al. 1996). The figure in Taiwan is lower than the $10.1 \%$ reported in our study. Different sampling frames and disorder diagnostic criteria should be taken into consideration when comparing these figures; for example, our study recruited patients with recurrent MDD through clinical settings while the cross-national epidemiological survey studied community samples.

Despite the sampling differences, we found that the clinical features of co-morbidity are generally consistent in China with those reported elsewhere in the world: co-morbidity is associated with increased MDD severity and chronic course, suicidal attempts, higher rates of neuroticism, and increased familial aggregation (Coryell et al. 1992; Kessler et al. 1998, 2003; Fava et al. 2000, 2004; Sareen et al. 2005; Bolton et al. 2008; Hettema, 2008; Howland et al. 2009; Lamers et al. 2011). The similarity we observed between comorbidity in China and the rest of the world encourages us to believe that our other findings will be applicable elsewhere. The diagnoses of MDD and comorbid anxiety disorders in our sample are lifetime. In a large clinical study, the current co-morbidity rate of any anxiety disorder was found to be $64 \%$ among current MDD patients, whereas the co-morbidity rate of lifetime diagnoses was 95\% (Brown et al. 2001). Hence we expect a large proportion of our patients with lifetime co-morbidity also would have had concurrent co-morbidity. However, this cannot be proved with our data.

Our second important finding concerns the heterogeneity of co-morbid anxiety disorders. Our large data set allowed us to ask whether co-morbidity between MDD and one anxiety disorder is the same as comorbidity between MDD and other anxiety disorders: for example, whether patients with co-morbid GAD look more or less similar in their clinical presentation and known risk factors for MDD to patients with comorbid social phobia. Our results indicate that there is considerable heterogeneity, and that when we talk of co-morbid anxiety it would be best to mean by this distinct co-morbidities with specific anxiety disorders rather than generic co-morbidity across all the anxiety disorders.

Significant heterogeneity was observed for three of the six MDD features that were tested for association with co-morbid anxiety disorders. They are (i) the number of MDD diagnostic criteria, (ii) suicidal attempt and, (iii) neuroticism measure. When controlling for the effects of all seven co-morbid anxiety disorders, we found that GAD predicts earlier onset and a higher number of DSM-IV criteria; panic disorder predicts higher number of DSM-IV criteria; animal phobia predicts higher number of episodes. There are conflicting results about whether co-morbid anxiety disorders are risk factors for suicide attempt among MDD patients (Roy, 1993; Sareen et al. 2005; Claassen et al. 2007; Bolton et al. 2008; Brown et al. 2010; Perroud et al. 2010). Our findings suggest that some co-morbid anxiety disorders (i.e. GAD) increase the risk for suicidal attempt, whereas others (i.e. blood-injury phobia) may be protective perhaps because the disorder is associated with a fear of death. Hence, treating all types of anxiety disorder together when testing for association with suicidal attempt might counterbalance the opposite effects, resulting in insignificant findings. Studies examining the relationship between co-morbid panic disorder and suicidal attempt show that either panic disorder increases the risk for suicidal attempt (Claassen et al. 2007) or the association is insignificant (Placidi et al. 2000). Our results disagree with most previous findings in suggesting that co-morbid panic disorder is protective against suicidal attempt. However, if we apply a conservative correction for multiple testing then this observation becomes non-significant, so only further study of this question will determine if this finding in Chinese women represents a true or false-positive result. Neuroticism showed the clearest pattern of heterogeneity: the test of heterogeneity gave the most significant of all results $\left(p=6.64 \times 10^{-6}\right)$.

We hypothesize that examining anxiety disorders as a single class hides important heterogeneity in their association with MDD. Heterogeneity is likely to be due to two factors: (i) opposite effects among different anxiety disorders; and (ii) individual effects of different anxiety disorders. Evidence for the former comes from results on suicidal attempt. While we found that all seven co-morbid anxiety disorders were significantly associated with suicidal attempt when tested separately, the direction of the effect differs: some anxiety disorders increase the occurrence of suicide attempts (GAD), while others (panic and blood-injury phobia) are protective (even when controlling for all the other co-morbid anxiety disorders).

Evidence for the latter comes from results on the number of DSM-IV diagnostic criteria and neuroticism. One important source for heterogeneity lies in differing genetic susceptibilities to each co-morbid condition. Thus, while we know that there is a common genetic predisposition to MDD and anxiety (Bienvenu et al. 2001; Khan et al. 2005; Middeldorp 
et al. 2005; Kendler et al. 2007; Spinhoven et al. 2009; Rhebergen et al. 2011), we also know that there are some unique genetic effects on anxiety disorders. For example, some genetic effects are shared between neuroticism and anxiety disorders; others are unique. The pattern of what is shared and what is common is specific to each condition.

The findings from the study have implications for the recognition, diagnosis and possibly treatment of MDD patients with different co-morbid anxiety disorders. For instance, the presence of GAD should alert doctors that the MDD patient is likely to be of a more severe kind, indicated by earlier onset and more symptom criteria, with suicidal ideation. Hence intervention could be applied at an earlier stage to prevent more negative outcomes.

\section{Limitations}

The reported results should be interpreted in the context of five potential limitations.

First, we only studied female patients and the relevance of our results to male patients is unknown. Second, the criteria used for the diagnoses of comorbid disorders of MDD were lifetime; hence the diagnoses of the seven anxiety disorders might not be co-morbid as in they might not have occurred concurrently with the worst episode of MDD. Third, patients were recruited through clinical settings; hence representativeness of our findings to the general population in China and the rest of the world is unknown. Fourth, cases recruited in the study were patients with at least two episodes of MDD; hence when co-morbid anxieties were tested for association with the number of episodes data, the effects and significance of effects were likely to be reduced compared with samples that only fulfilled the clinical diagnoses of MDD without the additional recruitment criteria. This might also have implications for the results of the global heterogeneity test for multiple regression coefficients. Fifth, our study differs from traditional family studies of anxiety disorders which involve the personal interview of first-degree family members (Crowe et al. 1983; Fyer et al. 1990; Brown et al. 1993; Weissman et al. 1993). Given the size of our study, it was only feasible to collect family history information from the patient about first-degree family members. An extensive literature on the family history method indicates that, in general, it has low sensitivity but high specificity (Andreasen et al. 1977, 1986; Roy et al. 1996), although some evidence for bias has been detected (Kendler et al. 1991). Furthermore, due to the design of our study, we do not have patients with anxiety but without MDD; hence we cannot fully test the transmission of MDD and the anxiety disorders.

\section{Declaration of Interest}

None.

\section{References}

Andreasen NC, Endicott J, Spitzer RL, Winokur G (1977). The family history method using diagnostic criteria: reliability and validity. Archives of General Psychiatry 34, 1229-1235.

Andreasen NC, Rice J, Endicott J, Reich T, Coryell W (1986). The family history approach to diagnosis: how useful is it? Archives of General Psychiatry 43, 421-429.

APA (1994). Diagnostic and Statistical Manual of Mental Disorders. American Psychiatric Association:

Washington, DC.

Bienvenu OJ, Brown C, Samuels JF, Liang KY, Costa PT, Eaton WW, Nestadt G (2001). Normal personality traits and comorbidity among phobic, panic and major depressive disorders. Psychiatry Research 102, 73-85.

Bolton JM, Belik S-L, Enns MW, Cox BJ, Sareen J (2008). Exploring the correlates of suicide attempts among individuals with major depressive disorder: findings from the national epidemiologic survey on alcohol and related conditions. Journal of Clinical Psychiatry 69, 1139-1149.

Brown GW, Harris TO, Eales MJ (1993). Aetiology of anxiety and depressive disorders in an inner-city population. 2. Comorbidity and adversity. Psychological Medicine 23, 155-165.

Brown LA, Gaudiano BA, Miller IW (2010). The impact of panic-agoraphobic comorbidity on suicidality in hospitalized patients with major depression. Depression and Anxiety 27, 310-315.

Brown TA, Campbell LA, Lehman CL, Grisham JR, Mancill RB (2001). Current and lifetime comorbidity of the DSM-IV anxiety and mood disorders in a large clinical sample. Journal of Abnormal Psychology 110, 585-599.

Cerdá M, Sagdeo A, Johnson J, Galea S (2010). Genetic and environmental influences on psychiatric comorbidity: a systematic review. Journal of Affective Disorders 126, 14-38.

Claassen CA, Trivedi MH, Rush AJ, Husain MM, Zisook S, Young E, Leuchter A, Wisniewski SR, Balasubramani GK, Alpert J (2007). Clinical differences among depressed patients with and without a history of suicide attempts : findings from the STAR*D trial. Journal of Affective Disorders 97, 77-84.

Clayton PJ, Grove WM, Coryell W, Keller M, Hirschfeld R, Fawcett J (1991). Follow-up and family study of anxious depression. American Journal of Psychiatry 148, 1512-1517.

Coryell W, Endicott J, Winokur G (1992). Anxiety syndromes as epiphenomena of primary major depression: outcome and familial psychopathology. American Journal of Psychiatry 149, 100-107.

Crowe RR, Noyes R, Pauls DL, Slymen D (1983). A family study of panic disorder. Archives of General Psychiatry 40, 1065-1069.

Endicott J, Andreasen N, Spitzer RL (1975). Family History-Research Diagnostic Criteria. Biometrics Research, New York State Psychiatric Institute: New York. 
Eysenck HJ, Eysenck SBG (1975). Manual of the Eysenck Personality Questionnaire. Hodder and Stoughton: London.

Fava M, Alpert JE, Carmin CN, Wisniewski SR, Trivedi MH, Biggs MM, Shores-Wilson K, Morgan D, Schwartz T, Balasubramani GK, Rush AJ (2004). Clinical correlates and symptom patterns of anxious depression among patients with major depressive disorder in STAR*D. Psychological Medicine 34, 1299-1308.

Fava M, Rankin MA, Wright EC, Alpert JE, Nierenberg AA, Pava J, Rosenbaum JF (2000). Anxiety disorders in major depression. Comprehensive Psychiatry 41, 97-102.

Fyer AJ, Mannuzza S, Gallops MS, Martin LY, Aaronson C, Gorman JM, Liebowitz MR, Klein DF (1990). Familial transmission of simple phobias and fears. A preliminary report. Archives of General Psychiatry 47, 252-256.

Garvey MJ, Tollefson GD, Tuason VB (1987). Comparison of major depressions with and without panic attacks. Comprehensive Psychiatry 28, 65-67.

Gorwood P (2004). Generalized anxiety disorder and major depressive disorder comorbidity: an example of genetic pleiotropy? European Psychiatry 19, 27-33.

Grunhaus L, Pande AC, Brown MB, Greden JF (1994). Clinical characteristics of patients with concurrent major depressive disorder and panic disorder. American Journal of Psychiatry 151, 541-546.

Hettema JM (2008). The nosologic relationship between generalized anxiety disorder and major depression. Depression and Anxiety 25, 300-316.

Howland RH, Rush AJ, Wisniewski SR, Trivedi MH, Warden D, Fava M, Davis LL, Balasubramani GK, McGrath PJ, Berman SR (2009). Concurrent anxiety and substance use disorders among outpatients with major depression: clinical features and effect on treatment outcome. Drug and Alcohol Dependence 99, 248-260.

Kendler KS, Gardner CO, Gatz M, Pedersen NL (2007). The sources of co-morbidity between major depression and generalized anxiety disorder in a Swedish national twin sample. Psychological Medicine 37, 453-462.

Kendler KS, Gardner CO, Neale MC, Prescott CA (2001). Genetic risk factors for major depression in men and women: similar or different heritabilities and same or partly distinct genes? Psychological Medicine 31, 605-616.

Kendler KS, Neale MC, Kessler RC, Heath AC, Eaves LJ (1992). Major depression and generalized anxiety disorder. Same genes, (partly) different environments? Archives of General Psychiatry 49, 716-722.

Kendler KS, Prescott CA (2006). Genes, Environment, and Psychopathology: Understanding the Causes of Psychiatric and Substance Use Disorders, 1st edn. Guilford Press: New York.

Kendler KS, Prescott CA, Myers J, Neale MC (2003). The structure of genetic and environmental risk factors for common psychiatric and substance use disorders in men and women. Archives of General Psychiatry 60, 929-937.

Kendler KS, Silberg J, Neale M, Kessler R, Heath A, Eaves L (1991). The family history method: whose psychiatric history is measured? American Journal of Psychiatry 148, 1501-1504.

Kessler RC, Berglund P, Demler O, Jin R, Koretz D, Merikangas KR, Rush AJ, Walters EE, Wang PS (2003). The epidemiology of major depressive disorder: results from the National Comorbidity Survey Replication (NCS-R). Journal of the American Medical Association 289, 3095-3105.

Kessler RC, DuPont RL, Berglund P, Wittchen HU (1999). Impairment in pure and comorbid generalized anxiety disorder and major depression at 12 months in two national surveys. American Journal of Psychiatry 156, 1915-1923.

Kessler RC, Nelson CB, McGonagle KA, Liu J, Swartz M, Blazer DG (1996). Comorbidity of DSM-III-R major depressive disorder in the general population: results from the US National Comorbidity Survey. British Journal of Psychiatry Supplement 30, 17-30.

Kessler RC, Stang PE, Wittchen HU, Ustun TB, Roy-Burne PP, Walters EE (1998). Lifetime panic-depression comorbidity in the National Comorbidity Survey. Archives of General Psychiatry 55, 801-808.

Khan AA, Jacobson KC, Gardner CO, Prescott CA, Kendler KS (2005). Personality and comorbidity of common psychiatric disorders. British Journal of Psychiatry: Journal of Mental Science 186, 190-196.

Lamers F, van Oppen P, Comijs HC, Smit JH, Spinhoven P, van Balkom AJLM, Nolen WA, Zitman FG, Beekman ATF, Penninx BWJH (2011). Comorbidity patterns of anxiety and depressive disorders in a large cohort study: the Netherlands Study of Depression and Anxiety (NESDA). Journal of Clinical Psychiatry 72, 341-348.

Maier W, Minges J, Lichtermann D (1995). The familial relationship between panic disorder and unipolar depression. Journal of Psychiatric Research 29, 375-388.

Middeldorp CM, Cath DC, Van Dyck R, Boomsma DI (2005). The co-morbidity of anxiety and depression in the perspective of genetic epidemiology. A review of twin and family studies. Psychological Medicine 35, 611-624.

Perroud N, Uher R, Hauser J, Rietschel M, Henigsberg N, Placentino A, Kozel D, Maier W, Mors O, Souery D, Dmitrzak-Weglarz M, Jorgensen L, Kovacic Z, Giovannini C, Mendlewicz J, Zobel A, Strohmaier J, McGuffin P, Aitchison KJ, Farmer A (2010). History of suicide attempts among patients with depression in the GENDEP project. Journal of Affective Disorders 123, 131-137.

Pini S, Cassano GB, Simonini E, Savino M, Russo A, Montgomery SA (1997). Prevalence of anxiety disorders comorbidity in bipolar depression, unipolar depression and dysthymia. Journal of Affective Disorders 42, 145-153.

Placidi GP, Oquendo MA, Malone KM, Brodsky B, Ellis SP, Mann JJ (2000). Anxiety in major depression: relationship to suicide attempts. American Journal of Psychiatry 157, 1614-1618.

R Development Core Team (2011). R: A Language and Environment for Statistical Computing. R Foundation for Statistical Computing: Vienna, Austria (http://www. r-project.org/). Accessed 25 February 2011.

Reich J, Warshaw M, Peterson LG, White K, Keller M, Lavori P, Yonkers KA (1993). Comorbidity of panic and major depressive disorder. Journal of Psychiatric Research 27, Suppl. 1, 23-33.

Rhebergen D, Batelaan NM, de Graaf R, Nolen WA, Spijker J, Beekman ATF, Penninx BWJH (2011). The 7-year course of depression and anxiety in the general population. Acta Psychiatrica Scandinavica 123, 297-306. 
Roy A (1993). Features associated with suicide attempts in depression: a partial replication. Journal of Affective Disorders 27, 35-38.

Roy MA, Walsh D, Kendler KS (1996). Accuracies and inaccuracies of the family history method: a multivariate approach. Acta Psychiatrica Scandinavica 93, 224-234.

Sareen J, Houlahan T, Cox BJ, Asmundson GJG (2005). Anxiety disorders associated with suicidal ideation and suicide attempts in the National Comorbidity Survey. Journal of Nervous and Mental Disease 193, 450-454.

Spinhoven P, de Rooij M, Heiser W, Smit JH, Penninx BWJH (2009). The role of personality in comorbidity among anxiety and depressive disorders in primary care and specialty care: a cross-sectional analysis. General Hospital Psychiatry 31, 470-477.
Weissman MM, Bland RC, Canino GJ, Faravelli C, Greenwald S, Hwu H-G, Joyce PR, Karam EG, Lee C-K, Lellouch J, Lépine J-P, Newman SC, Rubio-Stipec M, Wells JE, Wickramaratne PJ, Wittchen H-U, Yeh E-K (1996). Cross-national epidemiology of major depression and bipolar disorder. Journal of the American Medical Association 276, 293-299.

Weissman MM, Wickramaratne P, Adams PB, Lish JD, Horwath E, Charney D, Woods SW, Leeman E, Frosch E (1993). The relationship between panic disorder and major depression. A new family study. Archives of General Psychiatry 50, 767-780.

Zimmerman M, Chelminski I (2003). Clinician recognition of anxiety disorders in depressed outpatients. Journal of Psychiatric Research 37, 325-333. 